Research Article

\title{
Protective Factors Modulate the Risk of Beta Amyloid in Alzheimer's Disease
}

\author{
Bin Zhou ${ }^{1},{ }^{1}$ Kenichiro Tanabe, ${ }^{1}$ Shinsuke Kojima, ${ }^{1}$ Satoshi Teramukai, ${ }^{2}$ \\ Masanori Fukushima, ${ }^{1}$ and The Alzheimer's Disease Neuroimaging Initiative \\ ${ }^{1}$ Translational Research Center for Medical Innovation, Foundation for Biomedical Research and Innovation, Kobe, Japan \\ ${ }^{2}$ Department of Biostatistics, Kyoto Prefectural University of Medicine, Kyoto, Japan \\ Correspondence should be addressed to Bin Zhou; zhoubin@tri-kobe.org
}

Received 5 December 2019; Revised 12 June 2020; Accepted 30 June 2020; Published 30 October 2020

Academic Editor: Norbert Kovács

Copyright $\odot 2020$ Bin Zhou et al. This is an open access article distributed under the Creative Commons Attribution License, which permits unrestricted use, distribution, and reproduction in any medium, provided the original work is properly cited.

\begin{abstract}
Aim. To identify the factors protecting Abeta-positive subjects with normal cognition (NC) or mild cognitive impairment (MCI) from conversion to Alzheimer's disease (AD). Methods. Subjects with MCI in the Alzheimer's Disease Neuroimaging Initiative (ADNI) database, with baseline data for neuropsychological tests, brain beta amyloid (Abeta), magnetic resonance imaging (MRI), APOE genotyping, and 18F-FDG-PET (FDG), were included for analysis. Results. Elevated brain amyloid was associated with a higher risk of conversion from MCI to $\mathrm{AD}(41.5 \%)$ relative to Abeta levels of $<1.231$ (5.5\%) but was not associated with conversion from $\mathrm{NC}$ to $\mathrm{AD}(0.0$ vs. $1.4 \%)$. In the multivariate Cox regression analyses, elevated Abeta increased the risk of $\mathrm{AD}$, while higher whole-brain cerebral glucose metabolism (CGM) assessed by FDG decreased the risk of AD in subjects with the same amount of Abeta. Even in the patients with heavily elevated brain amyloid, those with FDG > 5.946 had a lower risk of AD. ApoE4 carrier status did not influence the protective effect. Conclusion. Higher average CGM based on FDG modified the progression to $\mathrm{AD}$, indicating a protective function. The results suggest that the inclusion of this CGM measured by FDG would enrich clinical trial design and that increasing CGM along with the use of anti-Abeta agents might be a potential prevention strategy for $\mathrm{AD}$.
\end{abstract}

\section{Introduction}

Alzheimer's disease $(\mathrm{AD})$ is a progressive neurodegenerative disease that lacks an effective treatment. The pathogenesis proceeds for decades before the onset of symptoms. Current research shows that the annual conversion rate from mild cognitive impairment $(\mathrm{MCI})$ to $\mathrm{AD}$ is approximately 10.2 to $33.6 \%$ based on a systematic review by Ward et al. [1]. Many factors associated with the conversion from MCI to $\mathrm{AD}$ have been explored. Elevated beta amyloid (Abeta) levels in the brain or cerebrospinal fluid (CSF) increase the risk of converting from normal cognition (NC) or MCI to $\mathrm{AD}$ [2-4]. Currently, a positive amyloid status, apolipoprotein E4 (ApoE4) carrier status, and elevated brain Abeta are risk factors for $\mathrm{AD}$ [5-7] and are inclusion criteria in clinical trial designs [8-10]. Subjective cognitive complaints (SCC) may inform the risk for future cognitive decline and track progression of self-perceived decline, particularly in those along the $\mathrm{AD}$ trajectory [10]. However, one study also suggested that elevated Abeta alone may be insufficient to produce cognitive change in individuals at risk for $\mathrm{AD}$ and supports the use of multiple biomarkers to stage $\mathrm{AD}$ progression $[5,11]$. Some subjects with a positive amyloid status remained cognitively normal during long-term follow-up [5].

Obviously, some of the factors that mediate the synthesis of amyloid in the pathogenesis of $\mathrm{AD}$ and the factors that decrease the risk are not yet clear. Arterial spin labelling magnetic resonance imaging (ASL-MRI) and $18 \mathrm{~F}$ fluorodeoxyglucose positron emission tomography (FDG) have comparable diagnostic accuracy in $\mathrm{AD}$ [12]. Changes in the cerebral blood flow (CBF) and brain FDG occur in different brain regions in Abeta-positive subjects across the $\mathrm{AD}$ continuum compared with Abeta-negative NC subjects; however, another study suggested the changes may have been the result of methodological differences [13]. In patients with MCI, FDG demonstrated hypometabolism 
and a component in the precuneus [14]. Hypoperfusion in the right precuneus, inferior parietal lobule, and middle cingulate gyrus were associated with the conversion from $\mathrm{MCI}$ to $\mathrm{AD}$ [15]. Many studies have shown that reductions in FDG were associated with conversion to $\mathrm{AD}$, and the combination of FDG scanning with measurement of hippocampal volume resulted in $96 \%$ specificity and $92 \%$ sensitivity in the prediction of conversion $[16,17]$. However, other studies indicated that a positive FDG scan in people with MCI was of no clinical value in the early prediction of progression to AD dementia [18, 19]. Decreased ASL values, mainly in the parietal and frontotemporal areas, were shown in a group of patients with the first signs of cognitive instability [20]. Whether these changes in CBF and uptake of FDG, which are physiological factors, can modulate the progression to $\mathrm{AD}$ in Abeta-positive subjects is not yet clear.

We used the ADNI data, including the pathophysiological factors of brain Abeta measured by PET, brain structure by MRI, and cerebral glucose metabolism (CGM) by FDG to explore the factors that mediate the conversion to $\mathrm{AD}$ in Abeta-positive and Abeta-negative MCI and NC subjects. We did not include neuropsychological tests, to avoid circularity issues in prediction.

\section{Subjects and Methods}

2.1. Subjects. We included all subjects with $\mathrm{MCI}$ or $\mathrm{NC}$ in the ADNI 1 and ADNI GO/2 phases obtained from the publicly available data repository, the Alzheimer's Disease Neuroimaging Initiative (ADNI) database (adni.loni.usc.edu).

The primary goal of ADNI has been to test whether serial MRI, PET, other biological markers, and clinical and neuropsychological assessments can be combined to measure the progression of $\mathrm{MCI}$ and early $\mathrm{AD}$.

The data used in this article were downloaded from the ADNI website in May 2019, including ADNIMERGE and updated diagnosis information. The data included florbetapir-PET scans to obtain a measure of cerebral amyloidosis, FDG, UCSF structural MRI, APOE genotyping, and some cognitive tests. For up-to-date information on specific inclusion and exclusion criteria, please see http://adni.loni.usc.edu.

2.2. Psychometric Testing. The following measure was included in the analysis: mini-mental state examination (MMSE); we did not include other neuropsychological tests to avoid circularity issues related to the use of these tests for diagnosis.

2.3. Determination of Brain Amyloid Status by PET. Florbetapir-PET was conducted in accordance with the ADNI PET protocols, which are available online (http://adni.loni.usc .edu/data-samples/pet). Image processing was performed by the ADNI core laboratory as described by Landau et al. [2]. A PET scan was acquired 50-70 minutes after injection of florbetapir. Images were smoothed and aligned to an MPRAGE anatomic image to obtain cortical segmentation. A mean cortical standardized uptake value ratio (SUVr) in the lateral and medial frontal, anterior and posterior cingulate, lateral parietal, and lateral temporal regions was obtained. A cortical florbetapir uptake value of $\geq 1.11$ was considered "elevated" or "positive" for cortical Abeta [2]

2.4. FDG Measures. The methods used to assess CGM based on 18F-FDG-PET have been described online (http://adni.loni.usc.edu/methods/pet-analysis-method/petanalysis/). Further details on the quality control analyses and procedures to enhance uniformity and reduce variability in PET images across centers are provided by Joshi et al. [21]. Quantitative CGM maps were intensity normalized to average brainstem FDG uptake. The metaregion of interest (meta-ROI) included is the mean SUVr of the temporal, angular, and posterior cingulate gyri [22].

MRI was performed using standardized protocols on 1.5 T MRI scanners with 3D T1-weighted sequences optimized for the different scanners, as indicated by http://www .loni.ucla.edu/ADNI/Research/ [7]. All images were corrected for spatial distortion due to gradient nonlinearity and normalized for B1 nonuniformity (see also http://www .loni.ucla.edu/ADNI/Data/). MRI measurements were reconstructed with the software program FreeSurfer, as described in detail by Fischl et al. [7]. The average cortical thickness (TA), standard deviation of thickness (TS), surface area (SA), and cortical volume (CV) were calculated. The automated Hippocampus Multi-Atlas Propagation and Segmentation (HMAPS) method was also used to measure several structures, such as the hippocampal volume. The factors included the $\mathrm{CV}$, TA, and SA of the following regions: the left and right entorhinal cortices, left and right hippocampi, left and right inferior temporal lobes, left and right parahippocampi, and left and right superior temporal lobes. Measurements from the left and right olfactory lobes, left and right hippocampi, left and right parahippocampi, medial portion of the orbital frontal cortices, lateral portion of the occipital cortex, inferior temporal gyrus, temporal pole, and the isthmus of the cingulate cortex were averaged to form a meta-ROI thought to be sensitive to early $\mathrm{AD}$-related neurodegeneration.

APOE genotyping was performed as described on the ADNI website (http://adni.loni.usc.edu/data-samples/ genetic-data/).

2.5. Statistics. Two-tailed Student's $t$-tests with equal variances and Welch's $t$-test for unequal variance were used to assess differences in demographic characteristics between Abeta-positive and Abeta-negative subjects. Wilcoxon's rank sum test was used only for follow-up time. Fisher's exact test was used for category variables.

Cox proportional hazard regression models were used to identify the statistically significant variables used to explore the risk factors for $\mathrm{AD}$. For variable selection in multivariate Cox regression analysis, the backward elimination method was used with the removal criterion $P$ value $\geq 0.1$. Similarly, in the survival tree analyses, the entry criterion was $P$ value $<$ 0.15 . The multiple testing problem was not considered. The cut-off points of Abeta and FDG tests were generated by the survival tree at the first cut-off value. 
Statistical analyses were performed using SAS version 9.4 (SAS Institute, Inc. Cary, NC, USA) and R version 3.6.1.

\section{Results}

Baseline brain PET amyloid data were available for $424 \mathrm{MCI}$ and $265 \mathrm{NC}$ subjects. Of these, $188(44.3 \%)$ of the $424 \mathrm{MCI}$ subjects were Abeta positive while $52(19.6 \%)$ of the 265 NC subjects were Abeta positive. The mean age of the Abeta-negative group was younger than that of the Abetapositive group in both the MCI and NC subjects. Age and ApoE status were significantly different between the Abetanegative and Abeta-positive groups in both the MCI and NC subjects. The median follow-up period was 25.87 (range: 4.72-60.89), 32.76 (range: 5.54-60.07), 19.47 (range: 5.5147.67), and 22.13 (range: 5.05-48.46) months from baseline in the Abeta-positive and Abeta-negative subjects in the MCI and NC groups, respectively (Table 1).

3.1. Cut-Off Values Determined by Survival Tree Method. We compared several methods based on multivariate Cox regression analysis (median, ROC curve, and survival tree) to find the optimal cut-off values for Abeta and FDG. These were determined to be 1.231 and 5.946, respectively, by the survival tree method at the first point.

In the subjects with Abeta values greater than 1.231, a further cut-off point was found at 1.462 where those with Abeta values $>1.462$ had a higher risk of conversion to AD compared with those with Abeta values between 1.231 and 1.462 (Suppl. Figure 1).

3.2. Outcomes of MCI and NC Subjects Based on Brain Abeta. Of the $424 \mathrm{MCI}$ subjects, 91 converted to AD. Although those who were Abeta positive had a higher risk of converting to $\mathrm{AD}(78,41.5 \%)$ than those who were Abeta negative, the majority of Abeta-positive MCI subjects (110,58.5\%) had not converted over a median period of 25.87 months of follow-up. If we assume that all subjects who were Abeta positive could develop $\mathrm{AD}$ during long-term follow-up, this difference in speed of conversion should be noted. Of the $\mathrm{NC}$ subjects, the 3 who converted to $\mathrm{AD}$ came from the group of 213 Abeta-negative NC subjects, and there was no conversion to $\mathrm{AD}$ in the 52 Abeta-positive NC subjects (Figure 1). The survival curves showed that at the proper cut-off points, Abeta status is a better predictor of disease progression than FDG (not shown) (Suppl. Figure 2).

3.3. Risk and Protective Factors Associated with Conversion from $M C I$ or $N C$ to $A D$. We found a significant difference in higher average brain FDG values between the conversion and nonconversion groups in both Abeta-positive and Abeta-negative subjects. However, the factors intracranial volume, fusiform gyrus volume, thickness of the entorhinal cortex, and hippocampal volume were significantly different in the conversion and nonconversion groups only in the Abeta-positive subjects (Suppl. Table 1). The results of the univariate and multivariate Cox regression analyses showed that higher average brain FDG, thickness of the entorhinal cortex, and hippocampal volume were protective factors for
$\mathrm{AD}$, while higher brain Abeta and ApoeE4 carrier status increased the risk of conversion to $\mathrm{AD}$ (Table 2).

3.4. Risk Classification by Brain PET Abeta and FDG. Risk classification using the combination of brain Abeta and FDG indicated in the Cox regression analysis showed that the lowest risk group consisted of those subjects with Abeta $\leq 1.231$ and FDG > 5.946, and the subjects with the highest risk of conversion to $\mathrm{AD}$ were those with Abeta $>1.231$ and $\mathrm{FDG} \leq 5.946$. Even in the group with very highly elevated Abeta, greater than 1.462, the protective effect of higher FDG still remained; there was a large difference in the conversion rate for the higher and lower FDG groups. Following stratification by ApoE4 status, higher FDG still showed a protective effect in the different groups. In ApoE4 noncarriers, the hazard ratio for Abeta $>1.231$ and FDG $\leq 5.946$ was much higher than in ApoE4 carriers (Table 3 and Figure 2).

\section{Discussion}

Elevated brain Abeta indicated a higher risk of conversion from $\mathrm{MCI}$ to $\mathrm{AD}$; however, in the subjects with elevated brain Abeta, approximately $60 \%$ did not develop $\mathrm{AD}$ during the follow-up. The factors that mediated Abeta pathogenesis were ApoE4 and/or other factors. Our analysis found that increased CGM measured by FDG was associated with delayed progression to $\mathrm{AD}$ in both the Abeta-positive and negative subjects, indicating a protective function. Even in the subjects with heavy Abeta deposition, the protective effect still remained and was independent of the ApoE4 carrier status.

Cerebral glucose hypometabolism and low $\mathrm{CBF}$ have been reported in MCI and AD patients [12, 16-18]. Alterations in FDG have been observed at least 20 years before positive Abeta measurements. Subtle cognitive dysfunction has been observed at least 10 years before patients test positive for Abeta [23]. In familial AD, the cascade of Abeta, then altered metabolism and then atrophy is definitive [24]. Khosravi et al.'s study revealed FDG global quantification to be a superior indicator of cognitive performance in the $\mathrm{AD}$ and MCI patients compared to 18F-florbetapir-PET [25]. However, recent research has indicated that increased Abeta deposition leads to the progression to mild cognitive impairment but decreased glucose metabolism does not contribute to progression [26, 27]. Not all subjects with elevated Abeta in the brain show a decrease in CGM. Our analysis indicated that even in some subjects with heavily elevated Abeta, the CGM can still be high and that higher CGM may modulate the risk of Abeta causing progression to $\mathrm{AD}$, rather than being a diagnostic indicator.

Many papers have discussed unsuccessful trials in the $\mathrm{AD}$ field over the last 20 years [28-30] that focused on incorrect therapeutic targets and had methodological problems in the clinical trial design or began treatment too late. In these studies, the endpoint is a critical issue for clinical trial design [28]. However, we know that the inclusion criteria are also important because discrepancies between subjects can no doubt influence assessment of the efficacy of the interventions in 
TABLE 1: Baseline characteristics of subjects with brain PET Abeta.

\begin{tabular}{|c|c|c|c|c|}
\hline & \multicolumn{2}{|c|}{ MCI } & \multicolumn{2}{|c|}{$\mathrm{NC}$} \\
\hline & Abeta+, $N=188$ & Abeta-, $N=236$ & Abeta,$+ N=52$ & Abeta-, $N=213$ \\
\hline Age, mean (SD) & $73.6(6.7)$ & $70.2(7.7)^{*}$ & $75.3(5.8)$ & $72.4(5.9)^{*}$ \\
\hline \multicolumn{5}{|l|}{ Sex } \\
\hline Male & $109(58.0)$ & $123(52.1)$ & $16(30.8)$ & $103(48.4)^{*}$ \\
\hline Female & $79(42.0)$ & $113(47.9)$ & $36(69.2)$ & $110(51.6)$ \\
\hline MMSE, mean (SD) & $27.5(1.8)$ & $28.5(1.5)^{*}$ & $29.0(0.9)$ & $29.1(1.3)$ \\
\hline \multicolumn{5}{|l|}{ ApoE } \\
\hline 0 & $54(28.7)$ & $159(67.4)^{*}$ & $25(48.1)$ & $165(77.8)^{*}$ \\
\hline 1 & $100(53.2)$ & $67(28.4)$ & $25(48.1)$ & $43(20.3)$ \\
\hline 2 & $34(18.1)$ & $10(4.2)$ & $2(3.8)$ & $4(1.9)$ \\
\hline Follow-up time, median (range) & $25.87(4.72-60.89)$ & $32.76(5.54-60.07)^{*}$ & $19.47(5.51-47.67)$ & $22.13(5.05-48.46)$ \\
\hline
\end{tabular}

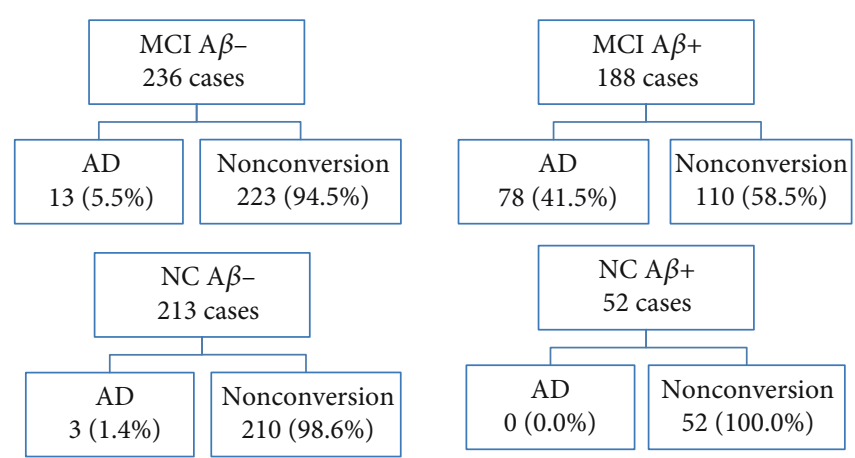

FIgURE 1: Outcomes of MCI and NC subjects based on PET brain Abeta. AD: Alzheimer's disease; NC: normal cognition; MCI: mild cognitive impairment; $\mathrm{A} \beta$ - and $\mathrm{A} \beta+$ : brain PET imaging Abeta negative and Abeta positive, respectively.

TABLE 2: The risk factors for conversion to AD by Cox regression.

\begin{tabular}{|c|c|c|}
\hline Factors & $\begin{array}{c}\text { Univariate } \\
\text { Hazard ratio }(95 \% \mathrm{CI})\end{array}$ & $\begin{array}{c}\text { Multivariate } \\
\text { Hazard ratio }(95 \% \mathrm{CI})\end{array}$ \\
\hline Hippocampal volume & $0.923(0.913-0.934)$ & $0.959(0.933-0.985)$ \\
\hline Average-brain FDG & $0.867(0.848-0.887)$ & $0.884(0.851-0.918)$ \\
\hline Cortical thickness of the entorhinal cortex & $0.903(0.887-0.918)$ & $0.941(0.903-0.981)$ \\
\hline Av45 & $1.468(1.359-1.585)$ & $1.244(1.113-1.391)$ \\
\hline Sex & $0.835(0.666-1.047)$ & $0.534(0.324-0.882)$ \\
\hline ApoE4 (2) & $4.502(3.191-6.352)$ & $2.063(0.996-4.276)$ \\
\hline$(1)$ & $2.892(2.267-3.690)$ & $2.024(1.135-3.608)$ \\
\hline$(0)$ & Reference & Reference \\
\hline Intracranial volume & $1.082(1.010-1.159)$ & \\
\hline Whole-brain volume & $0.997(0.996-0.998)$ & \\
\hline Fusiform volume & $0.805(0.765-0.846)$ & \\
\hline Ventricles & $1.013(1.009-1.018)$ & \\
\hline Age & $1.006(0.990-1.023)$ & \\
\hline Education & $0.971(0.934-1.010)$ & \\
\hline
\end{tabular}

${ }^{*} P<0.05$. Av45: brain PET amyloid; FDG: CGM based on 18F-FDG-PET.

these clinical trials. MCI subjects selected based on current technology are heterogeneous because their outcomes are very different, as shown in our analysis; this may have con- tributed to the failure of prevention and treatment in clinical trials. Both diagnosis and recruitment of appropriate trial participants are challenging. Targeting subjects with high 
TAble 3: Risk classification by brain Abeta and FDG stratified by ApoE.

\begin{tabular}{|c|c|c|c|c|}
\hline & Level & No. of events/no. of population & Hazard ratio & $95 \% \mathrm{CI}$ \\
\hline \multicolumn{5}{|l|}{ APOE- $\varepsilon 4$ carrier } \\
\hline \multirow{4}{*}{ a1. Group by AV45 and FDG } & A: AV45 $\leqq 1.231$ and FDG $>5.946$ & $4 / 103$ & (reference) & - \\
\hline & B: $A V 45 \leqq 1.231$ and $F D G \leqq 5.946$ & $2 / 18$ & 4.848 & $0.883-26.627$ \\
\hline & C: AV45 > 1.231 and FDG > 5.946 & $21 / 89$ & 6.798 & $2.333-19.808$ \\
\hline & D: AV45 > 1.231 and FDG $\leqq 5.946$ & $38 / 68$ & 22.601 & $8.031-63.605$ \\
\hline \multirow{2}{*}{ a2. $1.231<\mathrm{AV} 45 \leqq 1.462$} & A: FDG $\leqq 5.946$ & $18 / 35$ & 2.908 & $2.389-6.089$ \\
\hline & B: FDG $>5.946$ & $12 / 54$ & (reference) & - \\
\hline \multirow{2}{*}{ a3. AV45 > 1.462} & $\mathrm{~A}: \mathrm{FDG} \leqq 5.946$ & $20 / 33$ & 3.628 & $1.623-8.106$ \\
\hline & B: FDG $>5.946$ & $9 / 35$ & (reference) & \\
\hline \multicolumn{5}{|l|}{ APOE- $\varepsilon 4$ noncarrier } \\
\hline \multirow{4}{*}{ b1. Group by AV45 and FDG } & A: AV45 1.231 and FDG $>5.946$ & $5 / 272$ & (reference) & - \\
\hline & B: AV45 1.231 and $F D G \leqq 5.946$ & $5 / 39$ & 8.144 & $2.347-28.253$ \\
\hline & C: AV45 > 1.231 and FDG > 5.946 & $9 / 58$ & 7.206 & $2.356-22.039$ \\
\hline & D: AV45 > 1.231 and FDG $\leqq 5.946$ & $10 / 18$ & 50.72 & $17.007-151.257$ \\
\hline \multirow{2}{*}{ b2. $1.231<\mathrm{AV} 45 \leqq 1.462$} & A: FDG $\leqq 5.946$ & $3 / 10$ & 4.335 & $0.947-19.84$ \\
\hline & B: FDG $>5.946$ & $5 / 41$ & (reference) & - \\
\hline \multirow{2}{*}{ b3. AV45 > 1.462} & $\mathrm{~A}: \mathrm{FDG} \leqq 5.946$ & $7 / 8$ & 36945.00 & $0.00-$ missing \\
\hline & B: FDG $>5.946$ & $4 / 17$ & (reference) & \\
\hline
\end{tabular}

$P<0.001$. AV45: brain PET Abeta; FDG: CGM based on 18F-FDG-PET.

risk can reduce the sample size, but the reduction in variability across subjects could facilitate the efficacy assessment. Many studies have proposed methods to select subjects at high risk [31-33]; some of the proposed risk factors have included decreased cognition, measured by the Clinical Dementia Rating Scale Sum of Boxes (CDR-SOB), Alzheimer's Disease Assessment Scale-cognitive subscale (ADAS$\operatorname{cog}$ ), and auditory verbal learning test (AVLT), atrophy of the hippocampus and entorhinal cortex, and elevated brain Abeta and tau. Poor cognitive performance clearly implies disease progression. One of the limitations of these models is that the circularity of including neuropsychological tests in the diagnosis might mask the true risk and protective factors. Our analysis showed that the factors associated with pathophysiological processes were able to classify the risk of transition to $\mathrm{AD}$ very well in subjects positive for brain Abeta. Measurement of CGM by FDG combined with brain Abeta is suggested for the enrichment of clinical trial designs.

CGM as measured by FDG has been closely connected to neuronal activity. CGM can be used as a marker of synaptic dysfunction before an advance in neurodegeneration. In subjects with elevated brain Abeta, the altered metabolism measured by FDG was observed to reduce the progression of $\mathrm{AD}$. One hypothesis is that we can delay $\mathrm{AD}$ progression by increasing CGM.

In a previous study, six months of cognitive training increased CGM only in the MCI patients; however, there was no significant association between increased FDG uptake and improved cognition [34]. Physical activity has also been shown to alter brain glucose metabolism; however, these studies appear to be limited to conditions of high-intensity exercise in young and middle-aged cognitively normal cohorts. Aerobic training improved cognition and changed cerebral glucose metabolism in areas related to cognition in subjects with MCI [35]. Increased FDG uptake can also be due to an increase in CBF. Exercise training altered CBF and improved cognitive performance in older adults with and without cognitive impairment [36] and altered CBF and improved cognitive performance in older adults with and without cognitive impairment [37]. Further research including measurement of CBF is important, to clarify the relationship between $\mathrm{CBF}$, FDG uptake, and Abeta.

However, the majority of the identified studies in a recent review found no significant association between physical activity and the AD biomarkers Abeta 1-42, total tau, and phosphorylated tau in CSF, amyloid PET, hippocampal atrophy on MRI, and parietal temporal hypometabolism with brain FDG [38, 39]. In our analysis, the prognosis for Abeta-positive subjects was significantly different from those who were Abeta negative. The inconsistency between these results may be due to the differing backgrounds of the subjects whose Abeta information was unclear. Further research including the factors Abeta, CGM, and CBF would facilitate clarification of the protective effect of physical activity on $\mathrm{AD}$.

Because Abeta-targeted therapies have been mostly unsuccessful, researchers are becoming increasingly sceptical of the amyloid hypothesis and looking for other potential pathogeneses of AD. It has been observed that Abeta accumulation is 

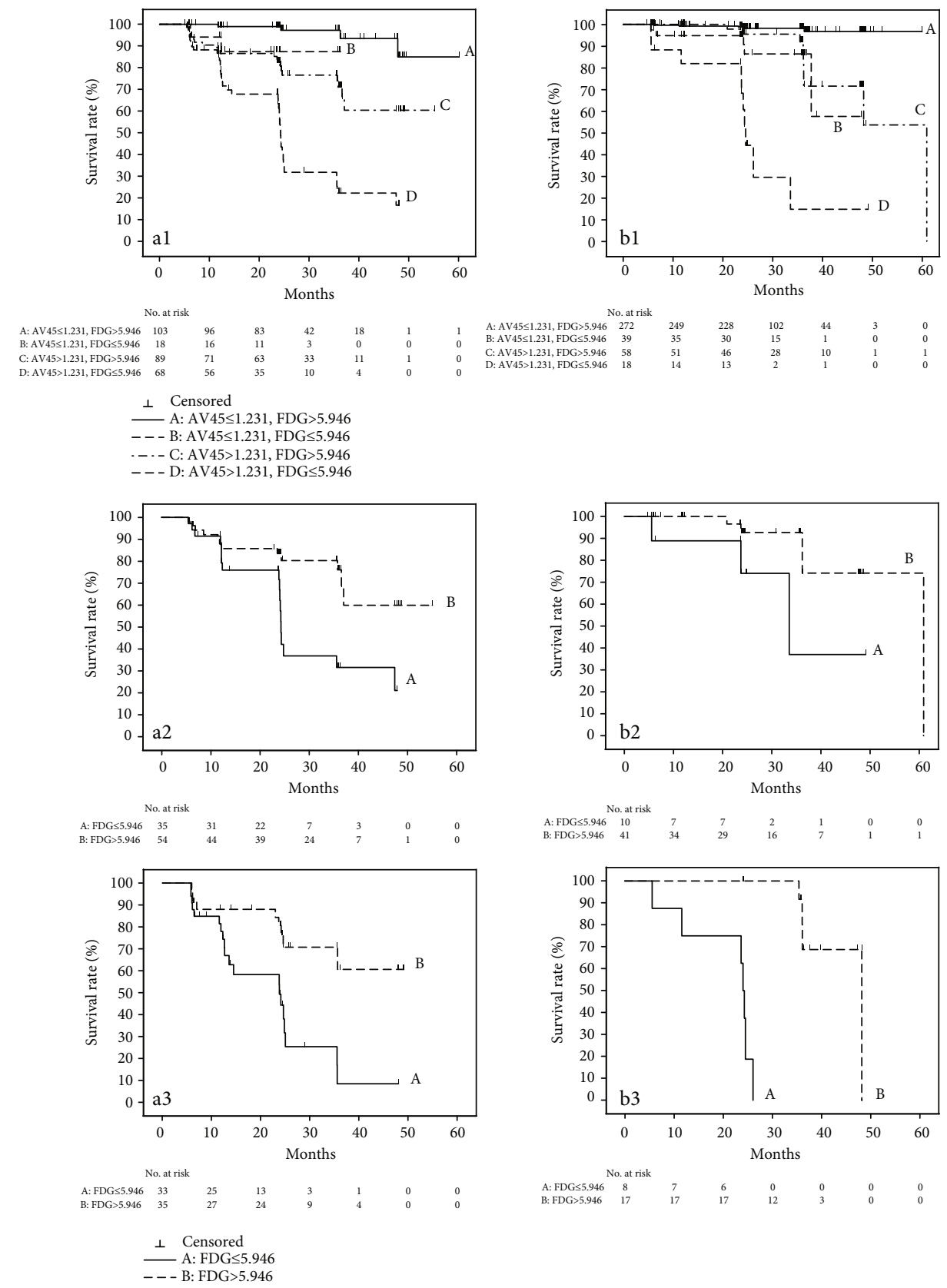

A: AV45 $\leq 1.231, \mathrm{FDG}>5.946$
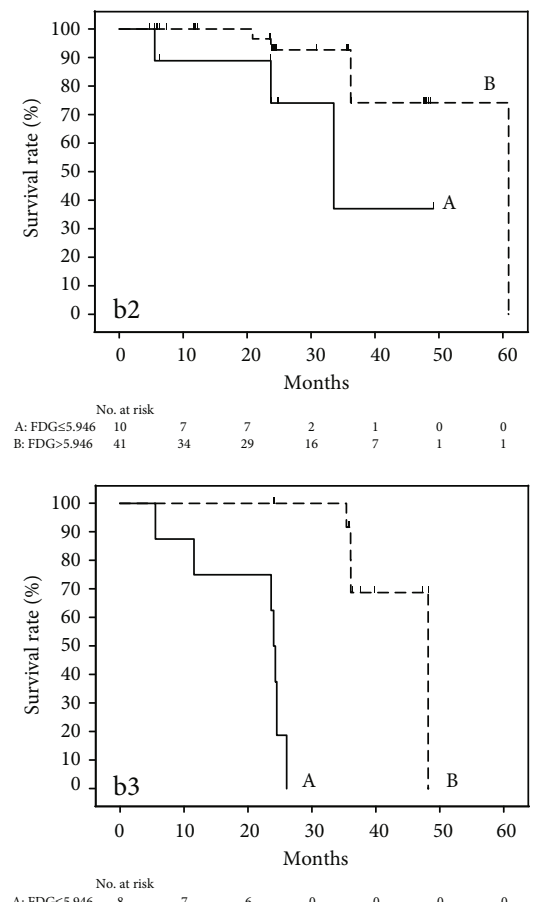

$\begin{array}{llllllll}\text { No. at risk } & & & & & & \\ \text { A: } \text { FDG } 5.946 & 8 & 0 & 0 & 0 & 0 \\ \text { B: FDG }>5.946 & 17 & 17 & 17 & 12 & 3 & 0 & 0\end{array}$

FIgURe 2: Risk classification by the combination of FDG with brain PET Abeta. (a) Survival curves by combination of Abeta and FDG in ApoE4 carriers. (a1, A) Abeta $\leq 1.231$ and FDG > 5.946; (B) Abeta $\leq 1.231$ and FDG $\leq 5.946$; (C) Abeta > 1.231 and FDG > 5.946; and (D) Abeta $>1.231$ and FDG $\leq 5.946$. (a2, A) $1.231<$ Abeta $\leq 1.462$ and FDG $\leq 5.946$ and (B) $1.231<$ Abeta $\leq 1.462$ and FDG > 5.946. (a3, A) $1.462<$ Abeta and FDG $\leq 5.946$ and (B) $1.462<$ Abeta and FDG $>5.946$. (b) Survival curves by combination of Abeta and FDG in non-ApoE4 carriers. (b1, A) Abeta $\leq 1.231$ and FDG $>5.946$; (B) Abeta $\leq 1.231$ and FDG $\leq 5.946$; (C) Abeta $>1.231$ and FDG > 5.946; and (D) Abeta $>1.231$ and FDG $\leq 5.946$. (b2, A) $1.231<$ Abeta $\leq 1.462$ and FDG $\leq 5.946$ and (B) $1.231<$ Abeta $\leq 1.462$ and FDG > 5.946. (b3, A) $1.462<$ Abeta and FDG $\leq 5.946$ and (B) $1.462<$ Abeta and FDG $>5.946$.

associated with tau aggregation, neuroinflammation, and synaptic dysfunction. In this study, elevated Abeta greatly increased the risk of $\mathrm{AD}$ in subjects with $\mathrm{MCI}$. Higher values of CGM measured by brain FDG decreased the risk of $\mathrm{AD}$ in Abeta-positive subjects, and this protection still remained even in subjects with very highly evaluated Abeta. Atrophy of the hippocampus and entorhinal cortex thickness are also strong risk factors for $\mathrm{AD}$. Our results with brain CGM confirmed that inclusion of these factors would enrich clinical trial design. Approaches designed to increase the physiological protective effect of CGM might be potential primary preventive interventions for $\mathrm{AD}$, along with anti-Abeta agents. Physical exercise or aerobic training still needs further research and data supporting a change in physiological factors, to confirm which exercise and its effect on the prevention of AD. 


\section{Data Availability}

The data used to support the findings are available from the ADNI database.

\section{Additional Points}

Data used in preparation of this article were obtained from the Alzheimer's Disease Neuroimaging Initiative (ADNI) database (adni.loni.ucla.edu). As such, the investigators within the ADNI contributed to the design and implementation of ADNI and/or provided data but did not participate in the present analyses or the writing of this report. A complete listing of ADNI investigators can be found at http://adni.loni.usc.edu/wp-content/uploads/ how_to_apply/ADNI_Acknowledgement_List.pdf.

\section{Conflicts of Interest}

The authors declare no conflicts of interest.

\section{Acknowledgments}

This research received funding from the Foundation for Biomedical Research and Innovation at Kobe.

\section{Supplementary Materials}

Supplementary Figure 1: survival tree to find the optimal cut-off point for AV45 and PDG. Supplementary Figure 2: survival curves by AV45 and FDG-PET. Supplementary Table 1: factors associated with conversion to AD stratified by brain PET Abeta. (Supplementary Materials)

\section{References}

[1] A. Ward, S. Tardiff, C. Dye, and H. M. Arrighi, "Rate of conversion from prodromal Alzheimer's disease to Alzheimer's dementia: a systematic review of the literature," Dementia and Geriatric Cognitive Disorders Extra, vol. 3, no. 1, pp. 320-332, 2013.

[2] S. M. Landau, M. A. Mintun, A. D. Joshi et al., "Amyloid deposition, hypometabolism, and longitudinal cognitive decline," Annals of Neurology, vol. 72, no. 4, pp. 578-586, 2012.

[3] C. R. Jack Jr., J. R. Barrio, and V. Kepe, "Cerebral amyloid PET imaging in Alzheimer's disease," Acta Neuropathologica, vol. 126, no. 5, pp. 643-657, 2013.

[4] M. C. Donohue, R. A. Sperling, R. Petersen et al., "Association between elevated brain amyloid and subsequent cognitive decline among cognitively normal persons," JAMA, vol. 317, no. 22, pp. 2305-2316, 2017.

[5] K. A. Johnson, A. Schultz, R. A. Betensky et al., "Tau positron emission tomographic imaging in aging and early Alzheimer disease," Annals of Neurology, vol. 79, no. 1, pp. 110-119, 2016.

[6] C. R. Jack, M. A. Bernstein, N. C. Fox et al., "The Alzheimer's Disease Neuroimaging Initiative (ADNI): MRI methods," Journal of Magnetic Resonance Imaging, vol. 27, no. 4, pp. 685-691, 2008.

[7] B. Fischl, M. I. Sereno, and A. M. Dale, "Cortical surface-based analysis. II: inflation, flattening, and a surface-based coordinate system," NeuroImage, vol. 9, no. 2, pp. 195-207, 1999.
[8] C. Frieden and K. Garai, "Structural differences between apoE3 and apoE4 may be useful in developing therapeutic agents for Alzheimer's disease," Proceedings of the National Academy of Sciences of the United States of America, vol. 109, no. 23, pp. 8913-8918, 2012.

[9] E. Cavedo, S. Lista, Z. Khachaturian et al., "The road ahead to cure Alzheimer's disease: development of biological markers and neuroimaging methods for prevention trials across all stages and target populations," The Journal of Prevention of Alzheimer's Disease, vol. 1, no. 3, pp. 181-202, 2014.

[10] A. Rizk-Jackson, P. Insel, R. Petersen, P. Aisen, C. Jack, and M. Weiner, "Early indications of future cognitive decline: stable versus declining controls," PLoS One, vol. 8, no. 9, article e74062, 2013.

[11] J. B. Toledo, for the Alzheimer's Disease Neuroimaging Initiative, M. W. Weiner et al., "Neuronal injury biomarkers and prognosis in ADNI subjects with normal cognition," Acta Neuropathologica Communications, vol. 2, no. 1, p. 26, 2014.

[12] E. S. Musiek, Y. Chen, M. Korczykowski et al., "Direct comparison of fluorodeoxyglucose positron emission tomography and arterial spin labeling magnetic resonance imaging in Alzheimer's disease," Alzheimer's \& Dementia, vol. 8, no. 1, pp. 5159, 2012.

[13] D. Tosun, N. Schuff, W. Jagust, M. W. Weiner, and for the Alzheimer"s Disease Neuroimaging Initiative, "Discriminative power of arterial spin labeling magnetic resonance imaging and $<$ sup $>18</$ sup $>$ F-fluorodeoxyglucose positron emission tomography changes for amyloid- $\beta$-positive subjects in the Alzheimer's disease continuum," Neurodegenerative Diseases, vol. 16, no. 1-2, pp. 87-94, 2016.

[14] I. Riederer, K. P. Bohn, C. Preibisch et al., "Alzheimer disease and mild cognitive impairment: integrated pulsed arterial spin-labeling MRI and18F-FDG PET," Radiology, vol. 288, no. 1, pp. 198-206, 2018.

[15] L. L. Chao, S. T. Buckley, J. Kornak et al., "ASL perfusion MRI predicts cognitive decline and conversion from MCI to dementia," Alzheimer Disease and Associated Disorders, vol. 24, no. 1, pp. 19-27, 2010.

[16] J. Ottoy, E. Niemantsverdriet, J. Verhaeghe et al., "Association of short-term cognitive decline and MCI-to-AD dementia conversion with CSF, MRI, amyloid- and 18F-FDG-PET imaging," NeuroImage: Clinical, vol. 22, p. 101771, 2019.

[17] L. Frings, S. Hellwig, T. Bormann, T. S. Spehl, R. Buchert, and P. T. Meyer, "Amyloid load but not regional glucose metabolism predicts conversion to Alzheimer's dementia in a memory clinic population," European Journal of Nuclear Medicine and Molecular Imaging, vol. 45, no. 8, pp. 1442-1448, 2018.

[18] N. Smailagic, M. Vacante, C. Hyde, S. Martin, O. Ukoumunne, and C. Sachpekidis, "18F-FDG PET for the early diagnosis of Alzheimer's disease dementia and other dementias in people with mild cognitive impairment (MCI)," Cochrane Database of Systematic Reviews, vol. 1, no. 1, article CD010632, 2015.

[19] L. M. Bloudek, D. E. Spackman, M. Blankenburg, and S. D. Sullivan, "Review and meta-analysis of biomarkers and diagnostic imaging in Alzheimer's disease," Journal of Alzheimer's Disease, vol. 26, no. 4, pp. 627-645, 2011.

[20] M. van der Thiel, C. Rodriguez, D. Van De Ville, P. Giannakopoulos, and S. Haller, "Regional Cerebral Perfusion and Cerebrovascular Reactivity in Elderly Controls With Subtle Cognitive Deficits," Frontiers in Aging Neuroscience, vol. 11, p. 19, 2019. 
[21] U. Joshi, P. G. H. M. Raijmakers, I. I. Riphagen, G. J. J. Teule, A. van Lingen, and O. S. Hoekstra, "Attenuation-corrected vs. nonattenuation-corrected 2-deoxy-2-[F-18] fluoro-D-glucosepositron emission tomography in oncology: a systematic review," Molecular Imaging and Biology, vol. 9, no. 3, pp. 99105, 2007.

[22] K. A. Johnson, R. A. Sperling, C. M. Gidicsin et al., "Florbetapir (F18-AV-45) PET to assess amyloid burden in Alzheimer's disease dementia, mild cognitive impairment, and normal aging," Alzheimer's \& Dementia, vol. 9, Supplement 5, pp. S72-S83, 2013.

[23] P. S. Insel, R. Ossenkoppele, D. Gessert et al., "Time to amyloid positivity and preclinical changes in brain metabolism, atrophy, and cognition: evidence for emerging amyloid pathology in Alzheimer's disease," Frontiers in Neuroscience, vol. 11, p. 281,2017

[24] B. A. Gordon, T. M. Blazey, Y. Su et al., "Spatial patterns of neuroimaging biomarker change in individuals from families with autosomal dominant Alzheimer's disease: a longitudinal study," The Lancet Neurology, vol. 17, no. 3, pp. 241-250, 2018.

[25] M. Khosravi, J. Peter, N. A. Wintering et al., "18F-FDG is a superior Indicator of cognitive performance compared to 18F-florbetapir in Alzheimer's disease and mild cognitive impairment evaluation: a global quantitative analysis," Journal of Alzheimer's Disease, vol. 70, no. 4, pp. 1197-1207, 2019.

[26] R. M. Anderson, C. Hadjichrysanthou, S. Evans, and M. M. Wong, "Why do so many clinical trials of therapies for Alzheimer's disease fail?,” The Lancet, vol. 390, no. 10110, pp. 23272329, 2017.

[27] S. Hatashita and D. Wakebe, "Amyloid $\beta$ deposition and glucose metabolism on the long-term progression of preclinical Alzheimer's disease," Future Science OA, vol. 5, no. 3, 2019.

[28] H. Oh, C. Madison, S. Baker, G. Rabinovici, and W. Jagust, "Dynamic relationships between age, amyloid- $\beta$ deposition, and glucose metabolism link to the regional vulnerability to Alzheimer's disease," Brain, vol. 139, no. 8, pp. 2275-2289, 2016.

[29] J. Cummings, "Lessons learned from Alzheimer disease: clinical trials with negative outcomes," Clinical and Translational Science, vol. 11, no. 2, pp. 147-152, 2018.

[30] P. S. Aisen, E. Siemers, D. Michelson et al., "What have we learned from EXPEDITION III and EPOCH trials? Perspective of the CTAD task force," The Journal of Prevention of Alzheimer's Disease, vol. 5, no. 3, pp. 171-174, 2018.

[31] J. D. Grill and S. E. Monsell, "Choosing Alzheimer's disease prevention clinical trial populations," Neurobiology of Aging, vol. 35, no. 3, pp. 466-471, 2014.

[32] B. Zhou, E. Nakatani, S. Teramukai, Y. Nagai, M. Fukushima, and Alzheimer's Disease Neuroimaging Initiative, "Risk classification in mild cognitive impairment patients for developing Alzheimer's disease," Journal of Alzheimer's Disease, vol. 30, no. 2, pp. 367-375, 2012.

[33] B. Zhou, Q. Zhao, S. Kojima et al., "One-year outcome of Shanghai mild cognitive impairment cohort study," Current Alzheimer Disease, vol. 16, no. 2, pp. 156-165, 2019.

[34] M. Medina, Z. S. Khachaturian, M. Rossor, J. Avila, and A. Cedazo-Minguez, "Toward common mechanisms for risk factors in Alzheimer's syndrome," Alzheimer's \& Dementia, vol. 3, no. 4, pp. 571-578, 2017.

[35] F. Henrique de Gobbi Porto, A. Martins Novaes Coutinho, A. Lucia de Sá Pinto et al., "Effects of aerobic training on cog- nition and brain glucose metabolism in subjects with mild cognitive impairment," Journal of Alzheimer's Disease, vol. 46, no. 3, pp. 747-760, 2015.

[36] T. Shah, G. Verdile, H. Sohrabi et al., "A combination of physical activity and computerized brain training improves verbal memory and increases cerebral glucose metabolism in the elderly," Translational Psychiatry, vol. 4, no. 12, article e487, 2014.

[37] A. J. Alfini, L. R. Weiss, K. A. Nielson, M. D. Verber, and J. C. Smith, "Resting cerebral blood flow after exercise training in mild cognitive impairment," Journal of Alzheimer's Disease, vol. 67, no. 2, pp. 671-684, 2019.

[38] L. A. van der Kleij, E. T. Petersen, H. R. Siebner et al., "The effect of physical exercise on cerebral blood flow in Alzheimer's disease," NeuroImage: Clinical, vol. 20, pp. 650-654, 2018.

[39] K. S. Frederiksen, L. Gjerum, G. Waldemar, and S. G. Hasselbalch, "Physical activity as a moderator of Alzheimer pathology: a systematic review of observational studies," Current Alzheimer Research, vol. 16, no. 4, pp. 362-378, 2019. 